\title{
Perspectiva temporal de los universitarios: implicaciones para la orientación educativa
}

\section{Florencia Teresita Daura}

orcid.org/00oo-0oo1-8101-6626

Universidad Austral, Argentina

fdaura@austral.edu.ar

\section{Resumen}

El presente estudio persigue una triple finalidad. Por un lado, evaluar la vinculación que existe entre la perspectiva temporal en la vida presente y la perspectiva futura trascendente en estudiantes de nivel superior, a partir de los resultados de la aplicación de dos cuestionarios diseñados por Zimbardo y Boyd. Por el otro, analizar la relación que estos factores temporales guardan con variables demográficas como la edad y el sexo, la pertenencia institucional y la práctica de una religión. Finalmente, de acuerdo con la información obtenida, brindar aportes sobre la acción orientadora del asesor académico y su influencia en el desarrollo del proyecto vital del alumno. En un primer momento, se desglosa un marco teórico sobre el constructo de la perspectiva temporal y sobre la acción orientadora del tutor; en segundo lugar, se describen y analizan los datos obtenidos en una muestra de 351 estudiantes argentinos pertenecientes a cuatro instituciones de educación superior; finalmente, se presentan algunas orientaciones pedagógicas que pueden llevarse a cabo en el espacio de la tutoría académica. Los datos recogidos muestran que existe una fuerte correlación entre los factores temporales y la manera de enfrentar el tiempo presente; en efecto, esta vinculación se produce entre los factores Pasado Negativo, el Presente Hedonista y Presente Fatalista, por un lado; y entre los factores Futuro, Futuro Trascendente y Presente Positivo. entre el presente positivo, el Futuro y el Futuro. Del mismo modo, se encontraron diferencias al vincular las dimensiones temporales con aspectos demográficos, principalmente con la institución de pertenencia y el sexo.

\section{Palabras clave}

Psicología de la educación; pedagogía universitaria; enseñanza superior; orientación escolar; asesoría académica (Fuente: Tesauro de la Unesco).

Recepción: 2016-06-21 | Envío a pares: 2017-05-18 | Aceptación por pares: 2017-07-25 | Aprobación: 2017-07-28 


\title{
The Time Perspective of University Students: Implications for Academic Guidance and Orientation
}

\begin{abstract}
The present study has three objectives. One is to assess the link that exists between the time perspective in present life and the transcendent future perspective in students of higher education, based on the results of the application of two questionnaires designed by Zimbardo and Boyd. It also is designed to analyze the relationship between these time factors and demographic variables such as age and gender, belonging to an institution, and the practice of a religion. Finally, pursuant to the information obtained, the study is intended to offer input on the orienting or guiding action of academic advisors and its influence on the development of a student's life project. First, a theoretical framework on the construct of the time perspective and on the guiding action of a tutor or advisor is broken down. Then, the information obtained from a sample of 351 Argentine students at four institutions of higher learning is described and analyzed. Finally, several educational guidelines are introduced that can be used in academic counselling. The data that was collected show a strong correlation between time factors and how to cope with present time. In effect, this link occurs between factors such as Negative Past, Hedonist Present and Fatalist Present, on the one hand; and between Future, Transcendent Future and Positive Present factors and Positive Present and Future factors, on the other. Similarly, differences were found when linking with dimensions with demographic aspects, mainly with belonging to an institution and gender.
\end{abstract}

\section{Keywords}

Educational psychology; university teaching; higher education; academic counselling (Source: Unesco Thesaurus). 


\section{Perspectiva temporal dos universitários: consequências para a orientação educativa}

\section{Resumo}

Este estudo tem uma tripla finalidade. Por um lado, avaliar a vinculação que existe entre a perspectiva temporal na vida presente e a perspectiva futura transcendente em estudantes de nivel superior, a partir dos resultados da aplicação de dois questionários elaborados por Zimbardo e Boyd. Por outro, analisar a relação que esses fatores temporais estabelecem com variáveis demográficas como idade, sexo, pertencimento institucional e prática de uma religião. Finalmente, de acordo com a informação obtida, oferecer contribuições sobre a ação orientadora do assessor acadêmico e sua influência no desenvolvimento do projeto vital do aluno. Num primeiro momento, desenvolve-se um referencial teórico sobre o construto da perspectiva temporal e sobre a ação orientadora do tutor; em segundo lugar, descrevem-se e analisam-se os dados obtidos numa amostra de 351 estudantes argentinos pertencentes a quatro instituições de ensino superior; finalmente, apresentam-se algumas orientações pedagógicas que podem ser realizadas no espaço da tutoria acadêmica. Os dados coletados mostram que existe uma forte correlação entre os fatores temporais e a maneira de enfrentar o tempo presente; de fato, essa vinculação é produzida entre os fatores passado negativo, presente hedonista e presente fatalista, por um lado; entre os fatores futuro, futuro transcendente e presente positivo, por outro. Do mesmo modo, encontraram-se diferenças ao relacionar as dimensões temporais com aspectos demográficos, principalmente com a instituição de pertencimento e o sexo.

\section{Palavras-chave}

Assessoria acadêmica; ensino superior; orientação escolar; pedagogia universitária; psicologia da educação (Fonte: Tesauro da Unesco). 
ISSN 0123-1294 | e-ISSN 2027-5358 | Educ.Educ. Vol. 20. No. 3 | Septiembre-diciembre de 2017 | pp. 403-418.

Universidad de La Sabana | Facultad de Educación

\section{La perspectiva temporal}

La perspectiva temporal (PT) es una dimensión cognitivo-motivacional que refiere a la percepción y vivencia del tiempo, capacidad característica del hombre, cuyo conocimiento es fundamental para comprender la conducta humana y, en particular, al estudiante universitario y su proyecto vital. El concepto fue introducido por Lewin (1935), quien lo comprende como la visión total que el individuo tiene de su pasado y futuro psicológico, existiendo en su presente. En la línea de Lewin, también Nuttin (1985) se ocupó del tema, señalando que los acontecimientos del pasado y los eventuales del futuro, en cuanto se hallan en el presente cognitivo, influyen en las motivaciones y conductas actuales, bajo la forma de recuerdos y expectativas. A partir de allí se efectuaron numerosas investigaciones sobre la temática en el campo de la psicología educativa, con diversos enfoques (Malas y Guiot, 2007).

En la presente investigación, se utiliza el modelo propuesto por Zimbardo y Boyd, quienes conciben la PT como "un proceso no consciente, mediante el cual el flujo de las experiencias personales y sociales se encuadran en categorías temporales que ayudan a dar orden, coherencia y significado a esas experiencias" (1999, p. 1271). Dichas categorías pueden ser modelos temporales cíclicos o eventos únicos en la vida, con los que se codifican, almacenan y resignifican los sucesos, las expectativas y las metas. Los autores proponen un modelo compuesto por cinco dimensiones temporales: Pasado Negativo, Pasado Positivo, Presente Hedonista, Presente Fatalista y Futuro, y sostienen que la PT puede centrarse en el pasado, en el presente o en el futuro,y modificarse e influir fuertemente sobre las acciones y las decisiones que se toman durante la vida.

Numerosos trabajos muestran la relación que existeentre la PTy distintas variables de estudio, como el rendimiento académico, la asunción de conductas positivas o negativas frente al estudio y a los retos en la actividad académica (Vázquez y Rapetti, 2006), la práctica de una religión (Ortuño, Paixão y Janeiro,
2013), la salud física y mental (Oyanadel y BuelaCasal, 2011), efectos de intervenciones pedagógicas (Oyanadel, Buela-Casal, Araa, Olivares y Vegal, 2014), entre otras, en las que se indica que el constructo puede modificarse por la influencia de factores externos, particularmente por la acción formativa de los educadores.

Boyd y Zimbardo (1997) también estudiaron la perspectiva temporal futura trascendente (PTFT) como otra dimensión de la experiencia temporal, que está conformada por las creencias que se poseen sobre la vida después de la muerte y que puede influir sobre las cogniciones y las acciones que se desarrollan durante la vida terrenal; no es exclusiva de los individuos que profesan una religión y se extiende a quienes procuran alcanzar un equilibrio vital (Ortuño et al., 2013). No obstante, existen pocas investigaciones sobre esta temática (Makhieva, 2012; Ortuño, Gomes, Paixão y Janeiro, 2012; Ortuño et al., 2013; Repišti, 2014; Seema, Sircova y Baltin, 2014), lo que puede deberse a la falta de consideración de la necesidad innata que tiene el hombre de trascenderse.

En lo que al campo educativo se refiere, interesa considerar cómo afecta la perspectiva temporal la concreción del proyecto vital del estudiante y, específicamente, cuáles son las acciones que el asesor académico puede realizar para que su labor orientadora sea más efectiva.

\section{El rol del docente como asesor académico en la universidad}

La función tutorial o de asesoría académica forma parte del rol del docente del nivel superior, quien a su vez se desempeña como investigador, gestor y enseñante. Detalles sobre su especificidad y sus características nos remiten a la antigüedad (Malbrán, 2004), en donde encontramos algunos ejemplos en los que el vínculo entre el maestro privado y el alumno se destacan por la acción modeladora ejercida por el primero, por el tiempo dedicado a la escucha y a 
la comprensión, por las enseñanzas transmitidas y por la huella dejada en el aprendiz, que se tradujeron luego en marcas imborrables en la historia de la humanidad. Así, en la antigua Grecia, se destaca la acción formativa brindada por Aristóteles a Alejandro Magno.

Con el surgimiento de la universidad la figura del tutor se formaliza, con el fin de brindar una "tutela formativa", transmitir el estilo propio de la universidad y resguardar la verdad científica en los estudiantes (Lázaro Martínez, 1997. p. 240). Con el paso del tiempo, si bien las funciones del asesor se redefinieron de acuerdo con el momento de la carrera en el que se encuentra el alumno y con el perfil a formar, muchas se han mantenido y otras se modificaron en coherencia con las competencias básicas que se deben desarrollar para desempeñar eficazmente el rol.

En correspondencia con las finalidades de la universidad de docencia, investigación y extensión, el asesor tiene que orientar al estudiante para que adquiera los conocimientos propios de la disciplina que a futuro desempeñará en el campo profesional $y$, junto con ello, procurar que adquiera otras competencias que son necesarias en su formación integral, en particular, las que coadyuvan al aprendizaje autónomo, a la concreción del proyecto personal de vida y, como derivación, a la selección de metas a corto, mediano y largo plazo que le permitan desarrollar todos los aspectos de su personalidad, trascendiéndose y realizando aportes en el contexto social en el que se encuentre.

Entre las acciones a realizar en el espacio de la tutoría en vínculo con el alumno, Cano González (2009) señala cuatro: facilitar la adaptación a la vida universitaria; orientar la concreción del proyecto vital, en el que tiene un lugar preponderante el desarrollo profesional; dar estrategias para mejorar el rendimiento académico; y facilitar el acceso al mundo laboral. Está claro que cada una de ellas puede concretarse a través de la aplicación de diversas estrategias; sin embargo, no es nuestro objetivo aquí detallaras. Por el contrario, sí podemos remarcar que todas se implementan para que el estudiante adquiera conocimientos y sabiduría, aspectos que hacen referencia al desarrollo de la virtud de la estudiosidad.

Esta virtud, que en el plano de la psicología cognitiva se refiere a la capacidad para autorregular el aprendizaje (Difabio de Anglat, 2011), trae consigo la sed de alcanzar la verdad y de ampliar los conocimientos. Es aquella que se pone de manifiesto en los alumnos que manifiestan interés por estudiar para adquirir nuevos aprendizajes, desarrollan el gusto por el estudio, son capaces de regular el esfuerzo y de buscar los medios para superar las dificultades que se presentan durante ese proceso, solicitan ayuda de otras personas que reconocen como portadoras de una mayor sabiduría y perseveran en la tarea, aunque esta les resulte dificultosa o ardua.

Por tratarse de una virtud, puede aumentar o disminuir, por lo que la guía del asesor académico es imprescindible. Es él quien debe ayudar a que el alumno se fije metas y priorice aquellas que son más importantes para su proyecto vital, y es quien tiene que facilitarle el autoconocimiento y las diversas herramientas con las que mejore su rendimiento académico, transmitiéndole la pasión por estudiar por medio de las actividades que él mismo realiza.

Este modelado (Bandura, 1986) también lo podrá efectuar sobre la forma particular con la cual visualiza su vida pasada, encara el presente y proyecta el futuro, ayudándolo a que adquiera lo que, a decir de Zimbardo y Boyd (1999), es una perspectiva temporal balanceada, caracterizada por el recuerdo de las vivencias positivas, la capacidad de disfrutar de la vida presente y de seleccionar metas que podrán concretarse en el porvenir. Es en ello en lo que puede ponerse en práctica la virtud de la prudencia que, en el proceso educativo, brinda los elementos necesarios para juzgar y decidir con equilibrio las cosas futuras (Martínez, 2003). 
A partir de estas consideraciones, el presente trabajo persigue tres objetivos generales:

- Evaluar la vinculación que existe entre la perspectiva temporal en la vida presente y la perspectiva futura trascendente en estudiantes de nivel superior, a partir de los resultados arrojados por la aplicación de dos cuestionarios diseñados por Zimbardo y Boyd (1999) y Boyd y Zimbardo (1997).

- Examinar la relación que los factores temporales guardan con la institución de pertenencia, el tipo de carrera, el sexo, la edad y la práctica religiosa de los estudiantes.

- Brindar aportes sobre la acción orientadora del asesor académico o del tutor y su influencia en el desarrollo del proyecto vital del alumno.

En el siguiente apartado se presenta el estudio efectuado con estudiantes de cuatro instituciones de educación superior que en forma voluntaria completaron tres cuestionarios.

\section{Metodología y procedimientos}

\section{Participantes}

La muestra se conformó con 351 estudiantes (180 mujeres y 171 varones con una media de 26 años, $D S=11,4)$ que durante 2013 y el 2014 cursaron carreras terciarias y universitarias de grado y de posgrado que se dictan en Argentina; 21 sujetos pertenecían a una institución de formación docente del interior del país (IFD1), 47 a otra organización similar de la Ciudad Autónoma de Buenos Aires (IFD2), 232 a una universidad privada de la provincia de Buenos Aires (UP1) y 47 a otra universidad privada localizada en la Ciudad Autónoma de Buenos Aires (UP2).

Tanto el IFD1 como el IFD2 reciben una población estudiantil que trabaja y pertenece a una clase social media y media-baja, a la que acompañan a través de diversos servicios (seguimiento personalizado, apoyo psicológico y/o fonoaudiológico y tutorías); también ofrecen una variedad de recursos pedagógico-didáctico (bibliotecas, laboratorio de informática, de idiomas, de físico-química, etc.). Se diferencian, en el primer caso, por tratarse de una organización de carácter privado y confesional que en sus carreras incluye materias teológicas, mientras en el segundo caso el instituto es estatal y aconfesional.

La UP1 y la UP2 son instituciones privadas y confesionales que dictan carreras universitarias y de posgrado. Brindan una formación científica, antropológica, espiritual y social, en la que consideran al estudiante como centro del proceso de enseñanzaaprendizaje. Los alumnos pertenecen a una clase sociocultural media y media-alta que, en su minoría, se desempeña laboralmente.

Con esta muestra se efectuó una investigación de carácter no experimental, descriptiva y correlacional, ya que las variables a analizar se observaron tal y como se dan en su contexto natural en un momento dado.

\section{Instrumentos de medición}

Cuestionario sociodemográfico. Se diseñó un cuestionario para recoger información referente al sexo, la edad, la institución, el tipo de carrera y la práctica religiosa de los sujetos.

Inventario de perspectiva temporal (Zimbardo Time Perspective Inventory, ZTPI). El inventario de respuestas tiene un formato de escala tipo Likert, con 5 alternativas de respuesta y 56 items que exploran cinco factores temporales claramente identificados (Zimbardo y Boyd, 1999): a) Pasado Negativo (PN) expresa el rechazo o la visión pesimista que se tiene sobre la vida pasada; b) Pasado Positivo (PP) evalúa los sentimientos positivos y de nostalgia hacia las experiencias vividas; c) Presente Hedonista (PH) muestra la medida en que el sujeto se orienta a buscar placer en el momento presente, con poca consideración de las consecuencias para el futuro; d) Presente Fatalista (PF) refleja una actitud de inde- 
fensión, desesperanza y resignación frente a la vida y al futuro; e) Futuro (F) expresa cómo se procura alcanzar objetivos y recompensas futuras.

Cuestionario sobre perspectiva temporal futura trascendente (Transcendental Future Time Perspective Scale, TFTPS). Es un instrumento unidimensional (Boyd y Zimbardo, 1997) que posee 10 items, con 5 opciones de respuesta. A partir del análisis factorial se excluyeron los ítems 4, 5, 7 y 10, por considerar que no se relacionan necesariamente con la convicción de que hay una dimensión del mundo y de la vida humana que trasciende las realidades estrictamente temporales. Efectivamente, a nuestro juicio, las personas pueden conceptuar como milagro lo que escapa a la comprensión de la regularidad natural, y considerar que las leyes de la ciencia no explican toda la realidad, porque tienen posiciones epistemológicas que no se limitan a la metodología de las ciencias positivas. Por otra parte, podrían sostener que hay en el hombre una dimensión espiritual, sin que ello se relacione necesariamente con un orden trascendente y, de modo análogo, no considerar la teoría darwiniana como opuesta al reconocimiento de dicho orden, ya que este juicio requiere un conocimiento especializado de las tesis y argumentos de la teoría, lo que introduciría un elemento de distorsión en la evaluación de las respuestas de los sujetos a dicho ítem. De hecho, en la validación de la escala, otros autores (Ortuño et al., 2013) han hallado que el ítem 5 presenta un valor de saturación de 0,15 en el factor, así como un bajo valor de comunalidad $\left(h^{2}\right.$ = 0,22), y su omisión eleva el alfa de Cronbach y la varianza total explicada. Asimismo, el ítem 7 registra un valor de saturación -0,50 más bajo que el resto de items, aunque aceptable.

\section{Procedimiento}

Se solicitó el permiso correspondiente en cada institución para aplicar las escalas y se brindó información a los participantes sobre los objetivos y el carácter confidencial y voluntario de su participación. Los instrumentos se administraron en forma colec- tiva en el aula durante un lapso que osciló entre 20 y 30 minutos. Los puntajes obtenidos se obtuvieron sumando las elecciones de los sujetos y convirtiendo la suma a escala 10 (el puntaje máximo posible se divide por la cantidad de ítems de la variable y se multiplica por diez). Para posteriores análisis, la muestra fue dividida en cuatro subgrupos por categorías de edad: hasta 21 años (231 sujetos), de 22 a 29 años (53 sujetos), de 30 a 39 años (52 sujetos) y de 40 o más años (49 sujetos). A su vez, las carreras fueron categorizadas en tres grupos que se denominaron teniendo en cuenta su área epistémica:

- Carreras técnicas: ingeniería industrial e ingenie-ría informática.

- Carreras humanístico-pedagógicas: licenciatura en psicología, licenciatura en psicopedagogía y profesorados del IFD1 y del IFD2.

- $\quad$ Carreras de economía: licenciatura en ciencias empresariales, agronegocios y contador público.

Los datos fueron procesados mediante la utilización del programa SPSS versión 13.0.

\section{Resultados}

\section{Análisis descriptivo del inventario de perspectiva temporal $y$ del cuestionario sobre perspectiva temporal futura trascendente}

A fin de hacer una primera caracterización de los estudiantes, se efectuó un análisis descriptivo con los valores arrojados por los instrumentos diseñados por Zimbardo y Boyd (1999) y Boyd y Zimbardo (1997) -media y desvío estándar- (Tabla 1). Los resultados expresan que la muestra que participó en el estudio posee una perspectiva temporal equilibrada, en la que predomina una visión positiva del pasado, un presente hedonista orientado a la satisfacción de necesidades sensibles, con bajo nivel de visión fatalista y un futuro positivo en el que se 
ISSN 0123-1294 | e-ISSN 2027-5358 | Educ.Educ. Vol. 20. No.3 | Septiembre-diciembre de 2017 | pp. 403-418.

Universidad de La Sabana | Facultad de Educación

pueden concretar metas vitales. Del mismo modo, coinciden con los de otras investigaciones (Ortuño y Gamboa, 2009; Worrell y Mello, 2007).

\section{Tabla 1. Valores descriptivos de los factores} del ZTPI y del TFTPS

\begin{tabular}{|c|c|c|c|}
\hline Factores & No. & Media & Desviación típica \\
\hline PN & 351 & 4,65 & 1,65 \\
\hline PP & 351 & 6,55 & 1,19 \\
\hline PH & 351 & 5,72 & 1,29 \\
\hline PF & 351 & 3,85 & 1,49 \\
\hline F & 351 & 6,94 & 1,23 \\
\hline FT & 351 & 5,61 & 2,40 \\
\hline
\end{tabular}

Fuente: Elaboración propia.

\section{Correlación entre los factores de la perspectiva temporal y la orientación futura trascendente}

Se calcularon los indices de correlación $r$ de Pearson entre los factores del ZTPI y el TFTPS (ver Tabla 2):

Tabla 2. Matriz de correlaciones entre los factores del ZTPI y el TFTPS

\begin{tabular}{|c|c|c|c|c|c|c|c|}
\hline & Factores & $\mathbf{1}$ & $\mathbf{2}$ & $\mathbf{3}$ & $\mathbf{4}$ & $\mathbf{5}$ & $\mathbf{6}$ \\
\hline 1. & $\begin{array}{c}\text { Pasado } \\
\text { Negativo }\end{array}$ & & 0,03 & $0,23^{* *}$ & $0,36^{* *}$ & 0,05 & 0,071 \\
\hline 2. & $\begin{array}{c}\text { Pasado } \\
\text { Positivo }\end{array}$ & & $0,22^{* *}$ & 0,05 & $0,23^{* *}$ & $0,15^{* *}$ \\
\hline 3. & $\begin{array}{c}\text { Presente } \\
\text { Hedonista }\end{array}$ & & & $0,38^{* *}$ & $-0,02$ & $-0,19^{* *}$ \\
\hline 4. & $\begin{array}{c}\text { Presente } \\
\text { Fatalista }\end{array}$ & & & & & $-0,04$ & $-0,18^{* *}$ \\
\hline 5. & Futuro & & & & & & $0,16^{* *}$ \\
\hline 6. & $\begin{array}{c}\text { Futuro } \\
\text { Trascendente }\end{array}$ & & & & & \\
\hline
\end{tabular}

Fuente: elaboración propia.

Se destacan las correlaciones positivas y significativas del Pasado Negativo con Presente Hedonista $r=(351)=0,23^{* *}, p=0,01$ y con Presente Fatalista $r=$ (351) $=0,36^{* *}, p=0,01$, en coincidencia con el estudio original de Zimbardo y Boyd (1999, p. 1276). Vale decir que, tanto en nuestra muestra como en el estudio original, se verificaría que los sujetos que tienen una vivencia negativa del pasado son más proclives a vivir el tiempo presente de un modo fatalista y hedonista, lo que expresa una lógica existencial, en este caso negativa.

El Pasado Positivo se correlaciona en forma positiva y significativa con el factor Presente Hedonista, $r=(351)=0,22^{* *}, p=0,01$; con el factor Futuro, $r=$ (351) $=0,23^{* *}, p=0,01$, en coincidencia con el estudio de Zimbardo y Boyd (1999) en el que los coeficientes son de $r=0,18^{* * *}$ y $r=0,12^{* * *}$ respectivamente; y con el factor Futuro Trascendente $r=(351)=0,15^{* *}, p=$ 0,01 , que en el estudio de Ortuño et al. (2013, p. 384) alcanza $r=0,17^{* *}$.

El Presente Fatalista se correlaciona en forma negativa y significativa con Futuro Trascendente, $r=$ $(351)=-0,18^{* *}, p=0,01$, en tanto que en el estudio de Ortuño et al. (2013, p. 384) la asociación es positiva, $r=0,20^{* *}$, lo que puede interpretarse como una diferencia en la concepción que tienen los sujetos de nuestra muestra en cuanto a la dimensión de trascendencia, no ligada a una visión determinista de la existencia. La correlación positiva y significativa del factor Presente Hedonista con el factor Presente Fatalista $r=(351)=0,38^{* *}, p=0,01$ complementaría esta visión: si el presente no está disponible a la libertad del sujeto, lo que queda es la búsqueda de satisfacciones inmediatas. Esta correlación, sin embargo, se verifica también en la muestra de Ortuño et al. (2013), $r=0,28^{* *}$, tanto como en la de Zimbardo y Boyd, $r=0,32^{* *}$.

El factor Futuro se correlaciona en forma positiva y significativa con el factor Futuro Trascendente, $r=(351)=0,16^{* *}, p=0,01$, en coincidencia con lo obtenido por Ortuño et al. (2013, p. 384), $r=0,14^{*}, y$ por Seema et al. (2014, p. 65), $r=0,114^{* *}$, lo que puede vincularse, nuevamente, con una visión de trascendencia compatible con la apertura al horizonte de futuro estrictamente temporal, que "permitiría dar orden, sentido y coherencia a toda la experiencia humana" (Ortuño et al., 2013, p. 385). 
Relación de las dimensiones de la perspectiva temporal y la perspectiva temporal futura trascendente con distintas variables demográficas

En todos los casos se efectuó un análisis de varianza (ANOva one way). Primeramente, se aplicó para comprobar si existen diferencias en función de la institución de pertenencia en los factores de la PT y en PTFT. Este análisis mostró que el grupo IFD1 alcanzó el mayor puntaje en el factor Presente Fatalista $(X=5,11)$ con diferencias significativas $[F$ $(1,351) 5,66, p>0,001$ ] en comparación con los valores medios de las demás instituciones. Asimismo, a UP2 tiene la media más alta $(X=6,50)$ en Futuro Trascendente $[F(1,351)=6,27, p>0,0001]$ (Figura 1).

Figura 1. Diferencias por institución en los factores de la PT y en FT

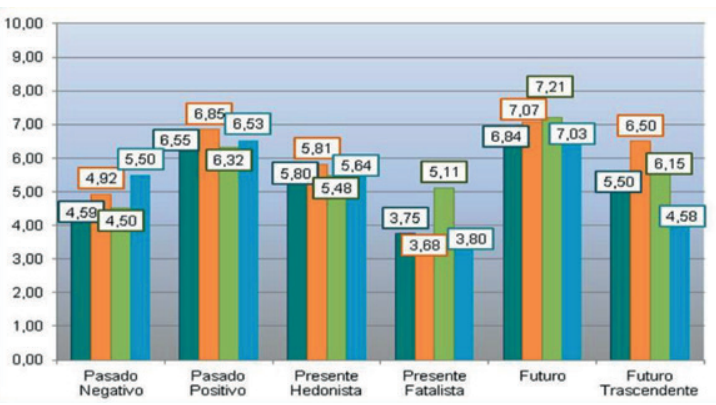

Fuente: Elaboración propia.

En las submuestras por tipos de carrera se encontraron diferencias significativas en el factor Presente Hedonista $[F(1,351)=12,592, p>0,0001]$ y en el factor Futuro Trascendente $[F(1,351)=30,206$, $p>0,0001]$ con medias más altas en las carreras de economía $(X=6,09)$ y pedagógico-humanísticas ( $X$ $=6,42)$ (Figura 2 ).

El análisis en función del sexo mostró que existen diferencias significativas a favor de las mujeres en Pasado Positivo $[F(1,351)=4,383, p>$ $0,003]$, Futuro $[F(1,351)=11,218, p>0,0001]$ y Futuro Trascendente $[F(1,351)=19,233, p>0,001]$. Los varones alcanzaron un puntaje significativamente mayor en Presente Hedonista $[F(1,351)=6,560, p>$
0,001 ] (Figura 3). Estos resultados coinciden con los encontrados en otros estudios (Boyd y Zimbardo, 1997; Díaz, 2006).

Figura 2. Diferencias por tipo de carrera en los factores de la PT y en FT

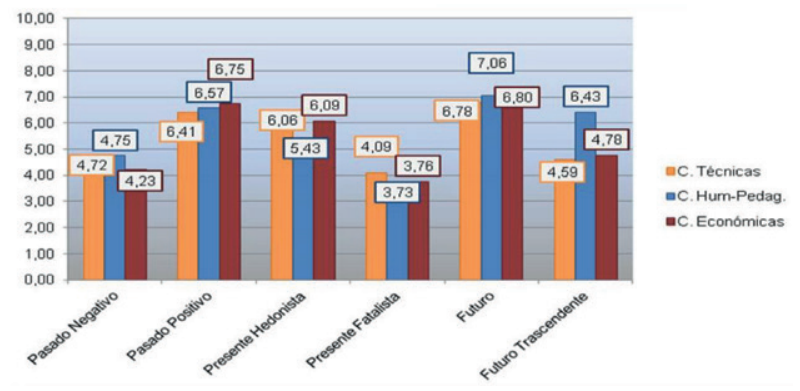

Fuente: Elaboración propia.

Figura 3. Diferencias por sexo en los factores de la PT y en FT

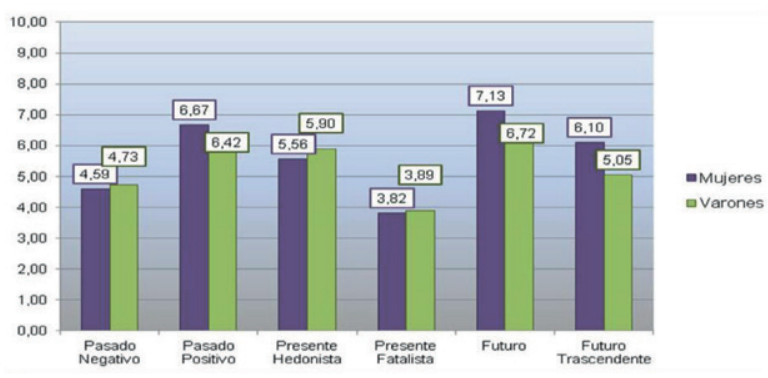

Fuente: Elaboración propia.

Las diferencias en función de las categorías de edad mostraron que los sujetos que tienen hasta 21 años poseen un mayor puntaje en el factor Presente Hedonista $(X=5,935)$ en comparación con los individuos de más de 40 años $(X=5,219)[F(1,351)$ $=6,063, p>0,001]$, resultados que coinciden con los de D'Alessio, Guarino, De Pascalis y Zimbardo (2003).

Se hallaron diferencias significativas en el factor Futuro Trascendente, con una media más alta para este último grupo $(X=7,32)$ en comparación con los demás $[F(1,351)=12,708, p>0,001]$ (Figura 4$)$. 
Figura 4. Diferencias por la categoría de edad en los factores de la PT y en FT

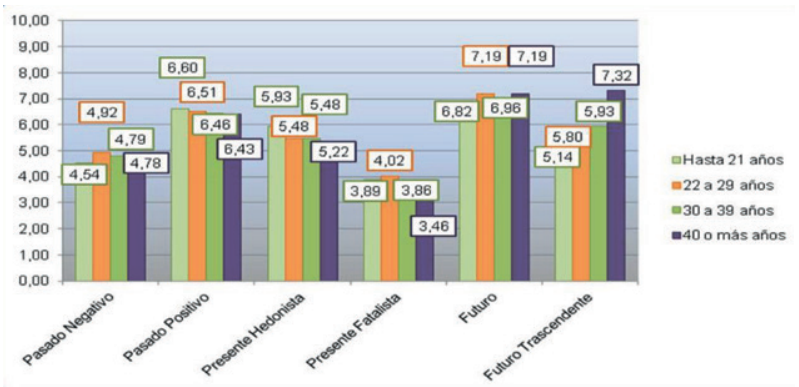

Fuente: Elaboración propia.

Se encontraron relaciones significativas entre la práctica de una religión y el factor FuturoTrascendente $[F(1,351)=172,89, p<0,01]$, con una media de 3,99 (SD $=1,29$ ) para el grupo no practicante, contra una media de $6,78(S D=1,15)$ para el grupo practicante; $y$ en el factor Presente Hedonista $[F(1,351)=6,16, p<0,02]$, con una media de 5,92 $(S D=1,29)$ para el grupo no practicante, contra una media de 5,55 $(S D=1,30)$ para el grupo practicante (Figura 5).

Figura 5. Comparacion del puntaje medio obtenido entre el factor Futuro Trascendente y la práctica de una religión

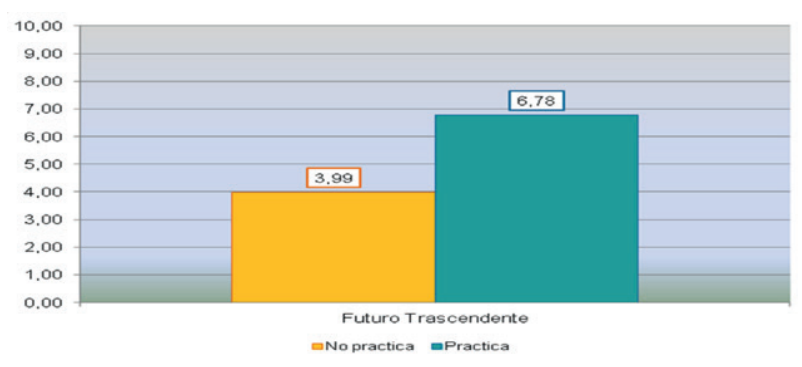

Fuente: Elaboración propia.

\section{Discusión y conclusiones}

El estudio del tiempo y, particularmente, la influencia que tiene en el desarrollo de la personalidad ocupa un lugar preponderante en el ámbito de la psicología. La persona vive en un momento histórico dado y percibe el transcurso de su ciclo vital de una forma determinada; sus pensamientos y comportamientos se modifican por el mayor o menor peso que tengan en ella la visión de las vi- vencias pasadas, presentes y futuras. Estas, sin ser determinantes, pueden modificarse por el efecto de la acción educadora, de los aprendizajes logrados y de cómo se resuelven las crisis normales del ciclo vital. Quien educa puede influir para que, quien aprende, llegue a tener una perspectiva equilibrada del tiempo, en la que recuerde prioritariamente las vivencias positivas del pasado, reelabore los recuerdos negativos; encare el presente de forma optimista, sin dejarse agobiar por las situaciones fatalistas; y visualice un futuro próspero, frente al cual puede anticiparse a partir de las acciones que elige realizar en el presente.

En línea con estas ideas, la presente investigación se planteó con los objetivos de: evaluar la vinculación existente entre la perspectiva temporal y la perspectiva temporal futura trascendente en estudiantes de nivel superior, a partir de los resultados arrojados por la aplicación de los cuestionarios diseñados por Zimbardo y Boyd (1999) y Boyd y Zimbardo (1997); examinar la relación que guardan los factores temporales con diversas variables demográficas; $y$, finalmente, brindar aportes sobre la acción orientadora que ejerce el asesor académico para influir positivamente en el desarrollo del proyecto vital del alumno.

El análisis de los datos recogidos muestra que existe una relación entre los factores Pasado Negativo, Presente Hedonista y Presente Fatalista en aquellos estudiantes que, por haber vivido experiencias negativas que los marcaron fuertemente, afrontan el presente entre dos polos, o bien en forma pesimista y desesperanzada o buscando la satisfacción de necesidades de orden sensible para evitar los recuerdos desagradables.

Asimismo, la asociación entre las dimensiones de Hedonismo y Fatalismo del presente es propia de los sujetos que viven el momento actual como sustraído a las decisiones propias, situación que tratan de compensar con la búsqueda de placeres sensibles inmediatos. Este efecto también estaría confirmado 
a partir de la correlación negativa hallada entre el factor Futuro Trascendente y el factor Presente Fatalista, resultado que aparece coherente con la relación teórica que se puede encontrar entre ambos tipos de vivencias, ya que sería esperable que los alumnos que tienen una cosmovisión trascendente posean la convicción de que el presente está en sus manos.

A la vez, la correlación positiva encontrada entre el futuro trascendente y la dimensión de futuro estrictamente temporal muestra que la capacidad para planificar metas a largo plazo está vinculada con la proyección del sentido de la vida más allá de los límites de la muerte. En este sentido, los alumnos que poseen un puntaje alto en ambos factores asocian la concreción de las metas factibles de alcanzar en la vida presente con el efecto que estas pueden tener en la vida posterrenal.

La existencia de una perspectiva temporal equilibrada se halló en la correlación existente entre los factores Pasado Positivo, Presente Hedonista y el Futuro, caracterizada por vivencias agradables del pasado, la capacidad para disfrutar el presente y para planificar metas a futuro. Esta perspectiva es la que permite que la persona afronte las vivencias diarias en forma balanceada, pudiendo disfrutar del quehacer diario; trate de encontrar soluciones a los inconvenientes que se presentan; confie en sus capacidades para lograrlo; y se perciba como responsable de su propia vida.

Los resultados referidos a la influencia de factores demográficos sobre las dimensiones de la perspectiva temporal mostraron diferencias significativas en la perspectiva futura trascendente en relación con la variable institución educativa, ya que la media en esta dimensión es sensiblemente más baja en la organización de gestión estatal, que no incluye en su proyecto educativo la formación humanístico-religiosa.

A la vez, las instituciones de formación docente del interior del país (IFD1) y de la Ciudad Autónoma de Buenos Aires (ISFD2) que reciben estudiantes con un nivel socioeconómico medio-bajo y poseen un proyecto educativo distinto difieren en la media de Futuro Trascendente. De modo análogo, la universidad privada de la provincia de Buenos Aires (UP1) y la de la provincia de Buenos Aires (UP2), que poseen alumnos con un nivel socioeconómico medio-alto, también registran esta diferencia. Estos efectos podrían interpretarse como un signo de la influencia que ejerce el contexto formativo institucional sobre la perspectiva extendida de futuro y el sentido trascendente de las experiencias vitales, con relativa independencia de las condiciones socioeconómicas.

Las diferencias halladas en la PT entre los sexos coinciden con estudios previos. En efecto, también en nuestra muestra las mujeres obtuvieron un puntaje mayor en los factores Pasado Positivo, Futuro y Futuro Trascendente, mientras que los varones solo alcanzaron un puntaje medio más alto en el factor Presente Hedonista, lo que permitiría hablar de la existencia de un perfil femenino y masculino transcultural, ligado a condiciones naturales, como la inclinación de la mujer a la contemplación y a la donación, en tanto que en el varón se acentúa la tendencia al placer sensible o a expresarla con mayor facilidad (Nuttin, 1985).

Las carreras de economía y las pedagógicohumanísticas mostraron diferencias significativas en los factores Presente Hedonista y Futuro Trascendente, respectivamente, resultados que son coherentes con el futuro perfil profesional de los graduados, que en un caso se orienta al desarrollo de negocios y operaciones financieras, mientras que en el otro se dirige a la formación de personas.

Las diferencias encontradas en función de la edad indican que la población de hasta 21 años alcanzó una puntuación mayor en Presente Hedonista, en coincidencia con el estudio de Omar et al. (2005), mientras que los sujetos con más de 40 años obtuvieron una puntuación más elevada en el factor 
Futuro Trascendente, hecho que podría responder a la madurez alcanzada y al replanteamiento del sentido de la vida. Efectivamente, los estudiantes que transitan la adolescencia se encuentran en una etapa del ciclo vital en la que se vivencian numerosos cambios en poco tiempo, lo cual genera una gran crisis que propicia la consolidación de la personalidad y el paso a la juventud. Es en ese proceso en el que se vislumbra la concreción de un proyecto de vida que supone un conjunto de metas que se plantean alcanzar (Carcelén y Martínez, 2008). Por el contrario, aquellos que están en la juventud o adultez se encuentran en etapas del ciclo vital en las que se definen, consolidan y redefinen las metas de vida elegidas en otro momento y donde se vivencian y elaboran varias crisis que Guardini (2015) denomina "de la experiencia", "de la experiencia de los límites" y de la "dejación".

Del mismo modo, las diferencias significativas encontradas entre el factor Futuro Trascendente y la práctica de una religión muestran la vinculación que existe entre la profesión de una creencia religiosa o filosófica y una cosmovisión que lleva al sujeto a plantearse metas terrenales que puedan dejar una huella en las generaciones venideras o en la vida después de la muerte.

Los resultados alcanzados pueden constituir un aporte para comprender cómo se vincula la perspectiva temporal con el modo en que el estudiante configura su personalidad, su existencia y, particularmente, cómo ello incide en su desarrollo académico y profesional. Es en el transcurrir de la vida cuando la personalidad se desarrolla y modifica, de acuerdo con el centro vital que la persona posee y con otros factores de orden hereditario y contextual. Al respecto, la madurez se consolida en la medida en que el hombre se conduce en forma intencional para alcanzar objetivos vitales, más aún cuando estos se dirigen a desarrollar todos los aspectos de su ser y le permiten salir de sí mismo y trascenderse (Nuttin, 1985).
Del mismo modo, la consideración de la dimensión trascendente del futuro permitiría entender de un modo cualitativamente diverso el significado que adquiere el tiempo de la vida en el cual se tiene la posibilidad de madurar, concretar proyectos $y$, junto con ello, llegar a un estadio relativo de plenitud, en el que se articulan el tiempo pasado y el futuro en el presente. De acuerdo con esto, si bien el hombre vive en el tiempo presente, es en el aquí y ahora donde proyecta su vida, proyección que remite al concepto de futuro. Al respecto, Heidegger (1924, p. 6) sostenía que "el ser futuro da tiempo, forma el presente y permite reiterar el pasado en el 'cómo' de su vivencia. [...] Esto significa que el fenómeno fundamental del tiempo es el futuro". Este mismo autor da un paso más, al considerar que el tiempo futuro tiene que ser comprendido a partir de la eternidad. Porque si la vida terrenal es finita, ¿cuál es el sentido de la existencia?, ¿con qué visión se concibe el tiempo vivido, ya en el pasado, y se afronta el presente? y, por último, ¿qué orientación se da al futuro y con qué finalidad se emprenden proyectos? El futuro, al ser desconocido, causa incertidumbre y duda; no obstante, el hombre puede imaginar y planificar lo que quiere realizar en el porvenir.

Estas reflexiones abren el camino para considerar algunas acciones que puede efectuar el asesor académico a fin de orientar al alumno en la concreción de su proyecto vital y para desarrollar una perspectiva temporal balanceada. Claro está que su labor tendrá que coordinarse con otros sectores de la institución académica a través de un plan de acción tutorial o de asesoramiento, tener en cuenta el perfil del estudiante a formar, y ello variará de acuerdo con el momento de la carrera en el que este se encuentre, de allí que su accionar puede estructurarse en tres momentos (Rodríguez, 2004): inicio de los estudios, curso de los estudios y final de los estudios. En cada uno de ellos, el tutor será el responsable de iniciar y tratar algunos temas que el estudiante continuará trabajando en pos de alcanzar su autonomía en cuatro áreas específicas: personal, familiar, académica y social (cfr. Tabla 3). 
Tabla 3. Temas a tratar en el asesoramiento académico

\begin{tabular}{|c|c|c|c|c|}
\hline $\begin{array}{c}\text { Área } \\
\text { Momento }\end{array}$ & Personal & Familiar & Académica & Social \\
\hline $\begin{array}{c}\text { Inicio de los } \\
\text { estudios }\end{array}$ & $\begin{array}{c}\text { Lugar de residencia y/o procedencia } \\
\text { (más aún cuando el alumno se trasladó } \\
\text { de ciudad para iniciar los estudios } \\
\text { superiores). } \\
\text { Expectativas sobre la carrera y sobre la } \\
\text { universidad. } \\
\text { Realización de hobbies. } \\
\text { Habilidades en otras áreas personales. } \\
\text { Percepción del propio compromiso con el } \\
\text { estudio. } \\
\text { Raíz o centro por el cual se vive y se toman } \\
\text { las decisiones en la vida: principales } \\
\text { valores que se sostienen en la vida. }\end{array}$ & $\begin{array}{c}\text { Percepción del apoyo } \\
\text { brindado por la familia. } \\
\text { Influencia de la } \\
\text { familia en la elección } \\
\text { de la carrera y de la } \\
\text { institución. } \\
\text { Situaciones particulares } \\
\text { que se estén viviendo } \\
\text { en ella. } \\
\text { Características del grupo } \\
\text { familiar. } \\
\text { Lugar de residencia. }\end{array}$ & $\begin{array}{l}\text { Experiencia educativa previa: } \\
\text { institución de procedencia. } \\
\text { Promedio obtenido. } \\
\text { Fortalezas y debilidades } \\
\text { académicas. } \\
\text { Expectativas y temores vinculados } \\
\text { con la vida académica. } \\
\text { Asignaturas de mayor interés. } \\
\text { Trayectoria profesional-académica } \\
\text { previa y su vinculación con los } \\
\text { estudios que se inician. } \\
\text { Rendimiento académico: } \\
\text { estrategias motivacionales y } \\
\text { cognitivas para desarrollarlo. }\end{array}$ & $\begin{array}{l}\text { Pertenencia a diversos } \\
\text { grupos sociales } \\
\text { (clubes, asociaciones, } \\
\text { etc.). } \\
\text { Actividades realizadas } \\
\text { con amigos. } \\
\text { Grupos de } \\
\text { pertenencia. }\end{array}$ \\
\hline $\begin{array}{c}\text { Durante los } \\
\text { estudios }\end{array}$ & $\begin{array}{l}\text { Cumplimiento de expectativas al iniciar } \\
\text { los estudios. } \\
\text { Desarrollo de habilidades y competencias. } \\
\text { Sentimientos de pertenencia a la } \\
\text { institución. } \\
\text { Raíz o centro por el cual se vive y se toman } \\
\text { las decisiones en la vida: principales } \\
\text { valores que se sostienen en la vida. }\end{array}$ & $\begin{array}{l}\text { Percepción del apoyo } \\
\text { brindado por la familia. } \\
\text { Situaciones particulares } \\
\text { que se estén viviendo } \\
\text { en ella. } \\
\text { Lugar de residencia. }\end{array}$ & $\begin{array}{c}\text { Rendimiento académico. } \\
\text { Características, fortalezas y } \\
\text { debilidades. }\end{array}$ & $\begin{array}{c}\text { Vínculo con } \\
\text { compañeros de clase y } \\
\text { de otros cursos. } \\
\text { Participación } \\
\text { en actividades } \\
\text { curriculares y } \\
\text { extracurriculares } \\
\text { propuestas en la } \\
\text { institución. } \\
\text { Iniciativa para } \\
\text { proponer actividades. }\end{array}$ \\
\hline $\begin{array}{c}\text { Finalización } \\
\text { de los } \\
\text { estudios }\end{array}$ & $\begin{array}{l}\text { Cumplimiento de expectativas al iniciar } \\
\text { los estudios. } \\
\text { Desarrollo de habilidades y competencias. } \\
\text { Autoconcepto y autoestima. } \\
\text { Sentimientos de pertenencia a la } \\
\text { institución. } \\
\text { Proyecto personal de vida en vínculo con el } \\
\text { desarrollo profesional. } \\
\text { Expectativas y temores en relación con la } \\
\text { proyección laboral. } \\
\text { Raíz o centro por el cual se vive y se toman } \\
\text { las decisiones en la vida: principales } \\
\text { valores que se sostienen en la vida. }\end{array}$ & $\begin{array}{c}\text { Percepción del apoyo } \\
\text { brindado por la familia. } \\
\text { Situaciones particulares } \\
\text { que se estén viviendo } \\
\text { en ella. } \\
\text { Lugar de residencia. } \\
\text { Autonomía brindada } \\
\text { para desarrollarse } \\
\text { profesionalmente. }\end{array}$ & $\begin{array}{l}\text { Rendimiento académico. } \\
\text { Características, fortalezas y } \\
\text { debilidades. } \\
\text { Realización de pasantías. }\end{array}$ & $\begin{array}{c}\text { Vínculo con } \\
\text { compañeros de clase y } \\
\text { de otros cursos. } \\
\text { Contactos } \\
\text { profesionales. } \\
\text { Desarrollo } \\
\text { socioprofesional. }\end{array}$ \\
\hline
\end{tabular}

Fuente: elaboración propia de acuerdo con Rodríguez Espinar (2004).

Los tópicos que aquí se plantean pueden ampliarse y modificarse de acuerdo con el momento personal en el que el alumno se encuentre y los intereses que posea. La clave está en favorecer el autoconocimiento y el planteo de un centro vital que, por el ejercicio de las virtudes arquitectónicas de la sabiduría y de la prudencia (Martínez, 2003), brinde coherencia a las decisiones que se tomen y propicie el desarrollo integral de la personalidad (Vázquez, 2012), con una visión balanceada del espacio temporal. 
ISSN 0123-1294 | e-ISSN 2027-5358 | Educ.Educ. Vol. 20. No. 3 | Septiembre-diciembre de 2017 | pp. 403-418.

Universidad de La Sabana | Facultad de Educación

\section{Referencias}

Bandura, A. (1986). Social foundations of thought and action: A social cognitive theory. Nueva Jersey: Prentice-Hall.

Boyd, J. N. y Zimbardo, P. G. (1997). Constructing time after death: The Transcendental Future Time Perspective. Time and Society, 6(1), 35-54.

Cano González, R. (2009). Tutoría universitaria y aprendizaje por competencias. ¿Cómo lograrlo? Revista Electrónica Interuniversitaria de Formación del Profesorado, 12(1), 181-204.

Carcelén Velarde,Ma.C.y Martínez,U.P.(2008). Perspectiva temporal futura en adolescentes institucionalizados. Revista de Psicología, 26(2), 255-276.

D’Alessio, M., Guarino, A., De Pascalis, V. y Zimbardo, P. G. (2003). Testing Zimbardo's Stanford Time Perspective Inventory (STPI)-Short form. An italian study. Time \& Society, 12(2/3), 333-347.

Díaz Morales, J. F. (2006). Estructura factorial y fiabilidad del Inventario de Perspectiva Temporal de Zimbardo. Psicothema, 18(3), 564-570.

Difabio de Anglat, H. (2011). De la studiositas al aprendizaje autorregulado. Acto inaugural de las sesiones ordinarias de la Academia de Humanidades, Facultad de Filosofía y Letras, Universidad Nacional de Cuyo, Mendoza.

Guardini, R. (2015). Las etapas de la vida (7 ed.). Madrid: Palabra.

Heidegger, M. (1924). El concepto del tiempo. Conferencia ante la Sociedad Teológica de Marburgo. Recuperado de http://190.186.233.212/filebiblioteca/Ciencias\%2oSociales/Martin\%2OHeidegger\%2O-\%2OEl\%2oConcepto \%2ode\%2oTiempo.pdf

Lázaro Martínez, A. (1997). La acción tutorial de la función docente universitaria. Revista Complutense de Educación, 8(1), 233-252.

Lewin, K. (1935). A dinamic theory of personality. Nueva York: McGraw-Hill.

Lloret-Segura, S., Ferreres-Traver, A., Hernández-Baeza, A.yTomás-Marco, I. (2014). El análisis factorial exploratorio de los ítems: una guía práctica, revisada y actualizada. Anales de Psicología, 30(3) 1151-1169.

Malas, Z. y Guiot, D. (2007). La Perspective Future: Essai de clarification des construits et util possibles en comportement du consommateur [La Perspectiva Futura: prueba de depuración de costrucciones y utiidades en el comportamiento del consumidor]. Ponencia presentada en las Sextas Jornadas Normandas de Investigación sobre la Sociedad de Consumo y los Coonsumidores. Groupe ESC Rouen, Francia, marzo. Recuperado de http://ziad.malas.free.fr/ACTIVITES_DE_RECHERCHE_files/JNConso2007MalasGuiot.pdf

Malbrán, M. del C. (2004). La tutoría en el nivel universitario. Revista de Informática Educativa y Medios Audiovisuales, 1(1), 5-11. 
Makhieva, L. (2012). The Transcendental-Future Time Perspective: past and future research [Abstract]. Sovremennye Issledovaniâ Social'nyh Problem, 6, 265-276.

Martínez, E. (2003). Educar en la virtud. Principios pedagógicos de Santo Tomás. Revista Electrónica Mensual del Instituto Universitario Virtual Santo Tomás, 1(1), 27-72.

Nuttin, J. (1985). Future Time Perspective and motivation: theory and research method. Hillsdale, NJ: Erlbaum.

Omar, A., Uribe Delgado, H., Aguiar de Souza, M. y Soares Formiga, N. (2005). Perspectivas de futuro y búsqueda de sensaciones en jóvenes estudiantes. Un estudio entre Argentina y Brasil. Revista Latinoamericana de Estudios Educativos, 35(1-2) 165-180.

Ortuño, V.y Gamboa, V. (2009). Estrutura fatorial do Zimbardo Time Perspective Inventory - ZTPI numa amostra de estudantes universitários portugueses. Avances en Psicología Latinoamericana, 27(1), 18-29.

Ortuño, V. E. C., Gomes, C., Paixão, Ma. P. B. A., Janeiro, I. (2012). Group differences in the Transcendental-Future Time Perspective: Portuguese results. Ponencia presentada en la Primera Conferencia Internacional sobre Perspectiva Temporal, Coimbra, Portugal, septiembre.

Ortuño,V.E.C.,Paixão,Ma.P.yJaneiro,I.N.(2013).Tempus post mortem? Adaptação portuguesa da TranscendentalFuture Time Perspective Scale (TFTPS). Avances en Psicología Latinoamericana, 37(2), 376-388.

Oyanadel, C.y Buela-Casal, G. (2011). La percepción del tiempo: influencias en la salud física y mental. Universitas Psychologica, 10(1), 149-161.

Oyanadel, C., Buela-Casal, G., Araa, T., Olivares, C. y Vegal, H. (2014). Percepción del tiempo: resultados de una intervención grupal breve para el cambio del perfil temporal. Suma Psicológica, 21(1), 1-7.

Repišti, S. (2014). A check of measurement characteristics of the Transcendental-Future Time Perspective Scale [Abstract]. Holon, 4(1)35-57. Recuperado de https://papers.ssrn.com/sol3/papers.cfm?abstract_id=2829139

Rodríguez Jaume, Ma. J. y Mora Catalá, R. (2001). Análisis factorial. En Estadística informática: casos y ejemplos con el SPSS (pp.127-142). Universidad de Alicante.

Rodríguez Espinar, S. (coord.) (2004). Manual de tutoría universitaria. Recursos para la acción. Barcelona: Octaedro/ICE Universidad de Barcelona.

Seema, R., Sircova, A. y Baltin, A. (2014). Transcendental future - Is it a healthy belief or a time perspective? The Transcendental-Future Time Perspective Inventory (TTPI) in estonian. Trames. A Journal of the Humanities and Social Sciences 18(68/63), 1, 57-75.

Vázquez, S. M. (2012). La filosofía de la educación. Estado de la cuestión y líneas esenciales (2 ed.). Buenos Aires: ciafic. 
ISSN 0123-1294 | e-ISSN 2027-5358 | Educ.Educ. Vol. 20. No. 3 | Septiembre-diciembre de 2017 | pp. 403-418.

Universidad de La Sabana | Facultad de Educación

Vázquez, S. M. y Rapetti, M. V. (2006). Future Time Perspective and motivational categories in argentinean adolescents. Adolescence, 41(163), 511-532.

Worrell, F. C. y Mello, Z. R. (2007). The reliability and validity of Zimbardo Time Perspective Inventory scores in academically talented adolescents. Educational and Psychological Measurement, 67(3), 487-504.

Zimbardo, P. G. y Boyd, J. N. (1999). Putting time in perspective: A valid, reliable individual differences metric. Journal of Personality and Social Psychology, 77, 1271-1288. 